\title{
Strong nuclear enhancement in intermediate mass Drell-Yan production
}

\author{
Jianwei Qiu ${ }^{a}$ and Xiaofei Zhang ${ }^{a, b}$ \\ ${ }^{a}$ Department of Physics and Astronomy, Iowa State University \\ Ames, Iowa 50011, USA \\ ${ }^{b}$ Physics Department, Kent State University \\ Kent, Ohio 44242, USA
}

(September 21, 2001)

\begin{abstract}
We calculate nuclear effect in Drell-Yan massive lepton-pair production in terms of parton multiple scattering in Quantum Chromodynamics (QCD). We present the nuclear modification to inclusive Drell-Yan cross section $d \sigma / d Q^{2}$ in terms of multiparton correlation functions. By extracting the size of the correlation functions from measured Drell-Yan transverse momentum broadening in nuclear medium, we determine the nuclear modification at $O\left(\alpha_{s} / Q^{2}\right)$. We find that the nuclear modification strongly enhances the inclusive DrellYan cross section in the intermediate mass region (IMR): $1.5<Q<2.5 \mathrm{GeV}$. We argue that the Drell-Yan process is responsible for the majority of the observed dimuon enhancement in the IMR in the $\mathrm{Pb}-\mathrm{Pb}$ collisions at CERN SPS.
\end{abstract}

PACS Numbers: 24.85.+p, 12.38.-t, 13.85.Qk, 25.40.Ve 
The inclusive Drell-Yan massive lepton-pair production in hadron-nucleus and nucleusnucleus collisions is an excellent laboratory for theoretical and experimental investigations of the role of nuclear medium in strong interaction dynamics. The massive lepton-pair production is also a channel for studying nuclear medium effect in production of quarkonium states and intermediate vector bosons. Recent measurements of massive lepton-pair production in both hadron-nucleus and nucleus-nucleus collisions show a very little nuclear dependence in Drell-Yan continuum, $d \sigma / d Q$, for lepton pair mass $Q$ larger than $4 \mathrm{GeV}$ [1.2]. Because of this lack of nuclear dependence in Drell-Yan continuum, the reported strong $\mathrm{J} / \psi$ suppression by the NA50 Collaboration was normalized to the Drell-Yan continuum in the lepton pair mass range $2.9<Q<4.5 \mathrm{GeV}$ [3].

Recently, it was shown by the NA38 and NA50 Collaborations that muon pair production for dimuon invariant mass between the $\phi$ and the $\mathrm{J} / \psi$ in heavy nucleus-nucleus collisions exceeds the expectation based on a linear extrapolation of the $p-A$ sources with the product of the mass numbers of the projectile and target nuclei [4]. The excess increases with the number of participant nucleons, and the ratio between the observed dimuon yield and the expected sources reaches a factor of 2 for central $\mathrm{Pb}-\mathrm{Pb}$ collisions. There have been a lot of effort to attribute such an excess to the sources other than the Drell-Yan continuum, such as the enhancement of open charm production [5], thermal dimuons production [6], and secondary meson-meson scattering in nuclear medium [7]. As shown in Ref. [4], the DrellYan continuum is the dominant source of the dilepton production in this relevant mass range in $\mathrm{Pb}-\mathrm{Pb}$ collisions with $158 \mathrm{GeV} /$ nucleon beam. An enhancement in the Drell-Yan continuum is much more effective than all other sources for interpreting the observed excess. Since the $\mathrm{J} / \psi$ cross section in $\mathrm{Pb}-\mathrm{Pb}$ collisions was normalized to the measured Drell-Yan continuum in the same experiment, it is important to understand the nuclear dependence of the Drell-Yan continuum in the same mass range [3]. It is the purpose of this Letter to calculate the nuclear modification to the Drell-Yan continuum in the mass range between the $\phi$ and the $\mathrm{J} / \psi$, which is reorganized as the intermediate mass region (IMR).

Since we are interested in the Drell-Yan continuum, or in general, Drell-Yan cross section at low invariant mass, $Q$, we ignore contributions from the intermediate vector boson $Z$. Therefore, the inclusive Drell-Yan cross section in a collision between hadrons, $h(p)+h^{\prime}\left(p^{\prime}\right) \rightarrow$ $\gamma^{*}(\rightarrow l \bar{l}(Q))+X$, can be expressed in terms of the cross section for production of an unpolarized virtual photon of the same invariant mass [8.9]

$$
\frac{d \sigma}{d^{4} Q}=\frac{1}{12 \pi^{3}} \frac{\alpha_{e m}^{2}}{S^{2} Q^{2}} W_{\mu \nu}(Q) P^{\mu \nu}(Q),
$$

where $S=\left(p+p^{\prime}\right)^{2}$ is the c.m. energy of the collision. The $W_{\mu \nu}(Q)$ in Eq. (1) is a hadronic tensor that satisfies electromagnetic (EM) current conservation, $Q_{\mu} W^{\mu \nu}(Q)=W^{\mu \nu}(Q) Q_{\nu}=$ 0 . Because of the EM current conservation of $W_{\mu \nu}(Q)$, the polarization tensor for the virtual photon, $P^{\mu \nu}(Q)=\sum_{\lambda=T, L} \epsilon_{\lambda}^{* \mu}(Q) \epsilon_{\lambda}^{\nu}(Q)$ in Eq. (11), is often replaced by $-g^{\mu \nu}$. The $T$ and $L$ here represent the transverse and longitudinal polarization of the virtual photon, respectively.

When energy exchange of the collision, $Q$, is much larger than the typical momentum scales of initial-state hadron wave functions, the Drell-Yan cross section, $d \sigma / d^{4} Q$, is dominated by a single hard collision between a quark and an antiquark from two incoming hadrons, as sketched in Fig. 1(a). Corrections due to interactions involving extra partons 
from incoming hadrons are power suppressed by the ratio of momentum scale of the hadron wave functions and the energy exchange of the hard collision. Therefore, leading power contribution to the Drell-Yan cross section can be factorized as [10]

$$
\frac{d \sigma_{h h^{\prime}}}{d^{4} Q} \approx \frac{d \sigma_{h h^{\prime}}^{(s)}}{d^{4} Q}=\sum_{a, b} \int d x \phi_{a / h}(x) \int d x^{\prime} \phi_{b / h^{\prime}}\left(x^{\prime}\right) \frac{d \hat{\sigma}_{a b}}{d^{4} Q},
$$

where superscript $(s)$ indicates a single hard scattering and $\sum_{a, b}$ run over all parton flavors. The $\phi$ 's are parton distributions and the factorization scale dependence are suppressed. The $d \hat{\sigma}_{a b} / d^{4} Q$ represents the perturbatively calculable rate of partonic collision. At the lowest order of quark-antiquark annihilation, the inclusive Drell-Yan cross section is given by [11]

$$
\frac{d \sigma_{h h^{\prime}}^{(s)}}{d Q^{2}} \approx\left(\frac{4 \pi \alpha_{e m}^{2}}{9 S Q^{2}}\right) \sum_{q} e_{q}^{2} \int d y \phi_{q / h}(x) \phi_{\bar{q} / h^{\prime}}\left(x^{\prime}\right)
$$

where the parton momentum fractions are given by

$$
x=\frac{Q}{\sqrt{S}} \mathrm{e}^{+y}, \quad \text { and } \quad x^{\prime}=\frac{Q}{\sqrt{S}} \mathrm{e}^{-y},
$$

with rapidity $y$. The high order corrections in powers of $\alpha_{s}$ to the inclusive cross section in Eq. (3) are well-defined in QCD perturbation theory [12]. For hadron-hadron collisions, Drell-Yan cross sections calculated in perturbative QCD are consistent with all existing data for $Q>4 \mathrm{GeV}$ 13.

In hadron-nucleus and nucleus-nucleus collisions, more partons are available at a given impact parameter. Consider the Drell-Yan process in hadron-nucleus collisions. Before the hard collision of producing the lepton-pair, partons from different nucleons can either interact between themselves, as sketched in Fig. 1)(b), or interact with the incoming parton, as sketched in Fig. 1(c). After taking a square of the scattering amplitude in Fig. 11(b), the leading contribution to the Drell-Yan cross section comes from the phase space where the parton momentum $k$ is pinched, which corresponds to the situation in which the interactions between partons from different nucleons took place "long" before the short-distance hard collision. That is, only a single parton from the nucleus participates the short-distance hard collision. The parton-parton interactions between nucleons are internal to the nucleus, and should not affect the dynamics of short-distance hard collision. Therefore, leading contributions from the multiple scattering process in Fig. 1 (b) to the Drell-Yan cross section should have the same factorized form as that in Eq. (2), except the parton distributions of a free hadron are replaced by the parton distributions of a nucleus. Those interactions internal to the nucleus in Fig. 1(b) are responsible for the phenomena of nuclear shadowing and EMC effect, which represent the differences between parton distributions of a free nucleon and those inside a nucleus. The nuclear shadowing and EMC effect provide small nuclear size dependence to the inclusive Drell-Yan cross section, or Drell-Yan continuum, $d \sigma / d Q$.

In this Letter, we concentrate on the contributions due to the type of parton multiple scattering shown in Fig. 1(c). Unlike the parton momentum $k$ in Fig. 1(b), the parton momentum $k^{\prime}$ in Fig. 1(c) is not pinched after we take a square of the scattering amplitude [14]. Therefore, the type of double scattering in Fig. 11(c) is a part of the hard collision for producing the lepton-pair. Because of the extra initial-state interaction, physical contributions 
from the double scattering are suppressed by a power of $Q^{2}$, known as the high twist contributions [15]. Although they are normally small in hadron-hadron collisions, such power suppressed contributions can be important in hadron-nucleus and nucleus-nucleus collisions, because of enhancement of nuclear size [16].

Before presenting our calculation of the nuclear modification to the Drell-Yan continuum caused by the type of multiple scattering in Fig. 1(c), we estimate the significance of such power suppressed modification. Let the inclusive Drell-Yan cross section in the collisions between nucleus $A$ and nucleus $B$ be approximated as

$$
\begin{aligned}
\frac{d \sigma_{A B}}{d Q^{2}} & \approx A B \frac{d \sigma_{N N}^{(s)}}{d Q^{2}}+\frac{d \sigma_{A B}^{(d)}}{d Q^{2}} \\
& \equiv A B \frac{d \sigma_{N N}^{(s)}}{d Q^{2}}\left[1+R_{A B}(Q)\right]
\end{aligned}
$$

where superscript $(d)$ represents the double scattering - a source of leading power corrections. The $d \sigma_{N N}^{(s)} / d Q^{2}$ is the single scattering cross section given in Eq. (3) with parton distributions of free hadron replaced by the effective parton distributions of a nucleon inside a nucleus. The $R_{A B}(Q)$ represents a ratio of the double scattering over the single scattering, and has the following dependence [16]

$$
R_{A B}(Q)=\frac{d \sigma_{A B}^{(d)} / d Q^{2}}{A B d \sigma_{N N}^{(s)} / d Q^{2}} \propto \frac{\alpha_{s}(Q)}{Q^{2}}\left\langle L_{A B}\right\rangle
$$

where $\left\langle L_{A B}\right\rangle \propto A^{1 / 3}+B^{1 / 3}$ is an effective medium length. Since $d \sigma / d Q=2 Q d \sigma / d Q^{2}$, the $R_{A B}(Q)$ in Eq. (6) is also equal to the ratio of the Drell-Yan continuum. If we assume that the $R_{A B}(Q)$ is positive and is about $5 \%$ at $Q=4 \mathrm{GeV}$ for the Drell-Yan continuum in hadron-nucleus collision, which is within the experimental uncertainty of dilepton yield at that energy [4], the ratio $R_{A B}(Q)$ will yield almost $50 \%$ enhancement to the Drell-Yan continuum in the central region of $\mathrm{Pb}-\mathrm{Pb}$ collisions at $Q=2 \mathrm{GeV}$. Such an enhancement could explain the majority of the dimuon excess observed in $\mathrm{Pb}-\mathrm{Pb}$ collisions.

In order to quantitatively understand the strong nuclear enhancement to the Drell-Yan continuum in the IMR, we have to address the following two questions: (1) can the leading power corrections to the Drell-Yan cross section be systematically calculated in QCD perturbation theory, and (2) is the sign of the nuclear modification to the Drell-Yan continuum positive. As we will show below, answers to these two questions are both positive.

As a direct consequence of the generalized factorization theorem [14], the leading power corrections to the inclusive Drell-Yan cross section or Drell-Yan continuum can be systematically calculated in QCD perturbation theory [15]. For the following derivation, we choose a frame in which the hadron (or nucleus) $A$ is moving along the $+z$-axis, and the $B$ is moving in $-z$ direction. We introduce the average nucleon momenta, $p \equiv P_{A} / A$ and $p^{\prime} \equiv P_{B} / B$. The \pm components of any vector $k$ are defined in terms of two lightlike vectors $n$ and $\bar{n}$ are $k^{+}=k \cdot n$ and $k^{-}=k \cdot \bar{n}$. These lightlike vectors are chosen such that $\bar{n} \cdot n=1$. In our choice of frame, the momentum $p$ is up to a mass term proportional to $\bar{n}$.

At the leading order in $\alpha_{s}$, the leading power corrections to the Drell-Yan cross section are given by the Feynman diagrams in Fig. 2, where the quark lines with a short bar are special quark propagators, which are equal to the normal quark propagators with the leading 
twist contributions removed [17]. According to the generalized factorization theorem, the leading power corrections to the Drell-Yan cross section in hadron-nucleus collisions can be factorized as [15],

$$
\begin{aligned}
W_{\mu \nu}^{(d)}(Q) & =\sum_{q} \int d x^{\prime} \phi_{\bar{q} / h^{\prime}}\left(x^{\prime}\right) \int d x d x_{1} d x_{2} \\
& \times T_{q A}\left(x, x_{1}, x_{2}\right) H_{\mu \nu}^{(d)}\left(x, x_{1}, x_{2} ; x^{\prime} ; Q\right),
\end{aligned}
$$

where $\sum_{q}$ runs over all quark and antiquark flavors. In the light-cone gauge of strong interaction, gluon field strength, $F^{+\alpha}\left(y^{-}\right)=n^{\rho} \partial_{\rho} A^{\alpha}\left(y^{-}\right)$, and the hadronic matrix element $T_{q A}\left(x, x_{1}, x_{2}\right)$ is given by 18

$$
\begin{aligned}
T_{q A}\left(x, x_{1}, x_{2}\right) & =\left(\frac{1}{x-x_{1}}\right)\left(\frac{1}{x-x_{2}}\right) \int \frac{d y^{-}}{2 \pi} \frac{d y_{1}^{-}}{2 \pi} \frac{d y_{2}^{-}}{2 \pi} \\
& \times \mathrm{e}^{i x_{1} p^{+} y^{-}} \mathrm{e}^{i\left(x-x_{1}\right) p^{+} y_{1}^{-}} \mathrm{e}^{i\left(x-x_{2}\right) p^{+} y_{2}^{-}} \\
& \times\left\langle P_{A}\left|\bar{\psi}(0) \frac{\gamma^{+}}{2} F^{+\alpha}\left(y_{2}^{-}\right) F_{\alpha}^{+}\left(y_{1}^{-}\right) \psi\left(y^{-}\right)\right| P_{A}\right\rangle .
\end{aligned}
$$

The partonic part $H_{\mu \nu}^{(d)}$ in Eq. (7) is given by the Feynman diagrams in Fig. 2, in which quark (antiquark) lines are contracted by $\frac{1}{2} \gamma \cdot p\left(\frac{1}{2} \gamma \cdot p^{\prime}\right)$ and gluon indices are contracted with $\frac{1}{2} d_{\alpha \beta}$. The transverse polarization tensor $d_{\alpha \beta}=-g_{\alpha \beta}+\bar{n}_{\alpha} n_{\beta}+n_{\alpha} \bar{n}_{\beta}$.

Because of EM current conservation, we can calculate the partonic hard part by contracting the $H_{\mu \nu}^{(d)}$ with either $-g^{\mu \nu}$ or the photon polarization tensor $P^{\mu \nu}(Q)$ in any gauge choice of EM interaction. Although the final answer is the same, contributions from different diagrams to the final answer are different. In addition, with an explicit polarization tensor, we can keep track the contributions to the different polarization states of the virtual photon. In the following, we present a result calculated in the light-cone gauge of EM interaction. In this gauge, the virtual photon's polarization tensor is given by [9]

$$
\begin{aligned}
P^{\mu \nu}(Q) & =-g^{\mu \nu}+\frac{Q^{\mu} n^{\nu}+n^{\mu} Q^{\nu}}{Q \cdot n} \\
& =2 P_{T}^{\mu \nu}(Q)+P_{L}^{\mu \nu}(Q) .
\end{aligned}
$$

With $Q^{\mu}=Q^{+} \bar{n}^{\mu}+Q^{-} n^{\mu}$ in our frame, we have

$$
P_{T}^{\mu \nu}(Q)=\frac{1}{2} d^{\mu \nu}, \quad \text { and } \quad P_{L}^{\mu \nu}(Q)=\frac{2 Q^{-}}{Q^{+}} n^{\mu} n^{\nu} .
$$

Contracting the partonic part $H_{\mu \nu}^{(d)}$ with the polarization tensors in Eq. (10), we find that only one diagram in Fig. 2(a) contributes to the longitudinally polarized virtual photon state, and two diagrams in Figs. 2(e) and (f) contribute to the transversely polarized virtual photon state. We obtain

$$
\begin{aligned}
H_{\mu \nu}^{(d)}(Q) P_{L}^{\mu \nu}(Q) & =e_{q}^{2}\left(\frac{1}{18}\right) \frac{g^{2}}{x x^{\prime}}(2 \pi)^{4} S \\
& \times \delta\left(Q^{+}-x p^{+}\right) \delta\left(Q^{-}-x^{\prime} p^{\prime-}\right) \delta^{2}\left(Q_{T}\right), \\
H_{\mu \nu}^{(d)}(Q) P_{T}^{\mu \nu}(Q) & =e_{q}^{2}\left(\frac{1}{18}\right) \frac{g^{2}}{x x^{\prime}}(2 \pi)^{4} S \\
& \times x\left[\frac{\delta\left(Q^{+}-x_{2} p^{+}\right)}{x_{2}-x_{1}}+\frac{\delta\left(Q^{+}-x_{1} p^{+}\right)}{x_{1}-x_{2}}\right] \delta\left(Q^{-}-x^{\prime} p^{\prime-}\right) \delta^{2}\left(Q_{T}\right),
\end{aligned}
$$


where $g$ is the coupling constant of strong interaction and $(1 / 18)$ is the overall color factor. Total partonic part to the inclusive Drell-Yan cross section is equal to the longitudinal contribution in Eq. (11) plus twice of the transverse contribution in Eq. (12). If we contract the $H_{\mu \nu}^{(d)}$ with $-g^{\mu \nu}$, we get the exact same total contributions, except they come from different diagrams.

As pointed out in Ref. [16], the perturbatively calculated hard part in the generalized factorization formula is independent of the structure - in particular the size - of the nuclear target. We need to find the medium size enhancement due to multiple scattering from the matrix element in Eq. (8). The $y_{i}^{-}$integrals in Eq. (8) parameterize the distance between the gluon and quark. Generally, the integrals over the distance $y_{i}^{-}$cannot grow with the size of the target because of oscillations of the exponential factors, unless the integrations of parton momentum fractions lead to vanishing exponential factors. This can be achieved if the integrations of parton momentum fractions are dominated by unpinched poles [16].

Combine the partonic parts in Eqs. (11) and (12) and the matrix element in Eq. (8), we find that the $d x_{1}$ and $d x_{2}$ integrations are dominated by the region where $x_{1} \sim x$ and $x_{2} \sim x$ from the poles. That the poles are in the matrix element $T_{q A}$ and do not have explicit $i \epsilon$ is due to our choice of light-cone gauge for the strong interaction. Within the light-cone gauge, we can either assign the $i \epsilon$ according the prescription introduced in the Appendix of Ref. [19] or carefully keep track of the $i \epsilon$ in the partonic part. For example, we left the following factor

$$
\left(\frac{x-x_{1}}{x-x_{1}+i \epsilon}\right)\left(\frac{x-x_{2}}{x-x_{2}-i \epsilon}\right)
$$

from the right-hand-side of Eq. (11). The numerator cancels the artificial poles in the matrix element and leaves the poles with correct $i \epsilon$ to the partonic hard part. We can also work in a covariant gauge of strong interaction without worrying about the artificial poles caused by the choice of light-cone gauge [18,20], and the same answer was derived.

Using the unpinched poles $1 /\left(x-x_{1}+i \epsilon\right)$ and $1 /\left(x-x_{2}-i \epsilon\right)$ to carry out the $d x_{1}$ and $d x_{2}$ integrations in Eq. (7), and substituting the hadronic tensor $W_{\mu \nu}^{(d)}$ into Eq. (1), we obtain the leading order medium size enhanced power corrections to the inclusive Drell-Yan cross section in hadron-nucleus collisions,

$$
\frac{d \sigma_{A h^{\prime}}^{(d)}}{d Q^{2}} \approx\left(\frac{4 \pi \alpha_{e m}^{2}}{9 Q^{2}}\right) \sum_{q} e_{q}^{2} \int d y\left(\frac{4 \pi^{2}}{3} \frac{\alpha_{s}}{Q^{2}}\right)\left[2 T_{q F}(x)-x \frac{\partial}{\partial x} T_{q F}(x)\right] \phi_{\bar{q} / h^{\prime}}\left(x^{\prime}\right)
$$

with $x$ and $x^{\prime}$ given in Eq. (四). The term proportional to $T_{q F}$ (its derivative) contributes to the longitudinally (transversely) polarized virtual photon state. The twist-4 quark-gluon correlation function $T_{q F}(x)$ is defined as [20,21]

$$
\begin{aligned}
T_{q F}(x) & =\int \frac{d y^{-}}{2 \pi} \mathrm{e}^{i x p^{+} y^{-}} \int \frac{d y_{1}^{-} d y_{2}^{-}}{2 \pi} \theta\left(-y_{2}^{-}\right) \theta\left(y^{-}-y_{1}^{-}\right) \\
& \times\left\langle P_{A}\left|\bar{\psi}(0) \frac{\gamma^{+}}{2} F^{+\alpha}\left(y_{2}^{-}\right) F_{\alpha}^{+}\left(y_{1}^{-}\right) \psi\left(y^{-}\right)\right| P_{A}\right\rangle .
\end{aligned}
$$

In deriving Eq. (13), we used the following approximation in the limit $x_{1} \rightarrow x$ and $x_{2} \rightarrow x$,

$$
\left[\frac{\delta\left(x_{2}-Q^{+} / p^{+}\right)}{x_{2}-x_{1}}+\frac{\delta\left(x_{1}-Q^{+} / p^{+}\right)}{x_{1}-x_{2}}\right]\left(\frac{1}{x-x_{1}+i \epsilon} \frac{1}{x-x_{2}-i \epsilon}\right)
$$




$$
\approx \delta^{\prime}\left(x-Q^{+} / p^{+}\right)\left(\frac{1}{x-x_{1}+i \epsilon} \frac{1}{x-x_{2}-i \epsilon}\right)+\ldots
$$

where "..." represents the terms that have less than two poles.

At the leading order in $\alpha_{s} / Q^{2}$, the medium enhanced power corrections to the inclusive Drell-Yan cross section in nucleus-nucleus collisions are given by

$$
\frac{d \sigma_{A B}^{(d)}}{d Q^{2}} \approx B \frac{d \sigma_{A N}^{(d)}}{d Q^{2}}+A \frac{d \sigma_{N B}^{(d)}}{d Q^{2}}
$$

with the nucleon-nucleus contributions given in Eq. (13).

In order to know the sign and magnitude of the medium enhanced power corrections, we need to know both the correlation function, $T_{q F}(x)$, and its derivative. Although the correlation function is nonperturbative, the generalized factorization theorem requires it to be a universal function. Therefore, we can find the information of $T_{q F}(x)$ from other observables.

In Ref. [21], Drell-Yan lepton-pair's transverse momentum broadening was calculated in terms of parton multiple scattering in perturbative QCD. It was found that the broadening is proportional to the same quark-gluon correlation $T_{q F}(x)$. Using data on Drell-Yan transverse momentum broadening in hadron-nucleus collisions, and a model for $T_{q F}(x)$ from Ref. [20],

$$
T_{q F}(x) \approx \lambda^{2} A^{4 / 3} \phi_{q / N}(x)
$$

it was found 21] that $\lambda^{2} \approx 0.01 \mathrm{GeV}^{2}$. The same model was also used to evaluate 2-jet momentum imbalance in photon-nucleus collision [20], and a larger value of $\lambda^{2}\left(\sim 0.05 \mathrm{GeV}^{2}\right)$ was extracted from Fermilab data [22]. Differences between these two observables were discussed in Ref. [16].

Taking the same model for the quark-gluon correlation function in Eq. (17), and substituting Eq. (16) into Eq. (6), we obtain

$$
\begin{aligned}
R_{A B}(Q) & \approx \frac{4 \pi^{2} \alpha_{s}}{3 Q^{2}} \lambda^{2} \\
& \times \frac{\sum_{q} e_{q}^{2} \int d y\left\{A^{1 / 3}\left[2 \phi_{q / N}(x)-x \frac{\partial}{\partial x} \phi_{q / N}(x)\right] \phi_{\bar{q} / N}\left(x^{\prime}\right)+B^{1 / 3}\left[x \leftrightarrow x^{\prime}\right]\right\}}{\sum_{q} e_{q}^{2} \int d y \phi_{q / N}(x) \phi_{\bar{q} / N}\left(x^{\prime}\right)},
\end{aligned}
$$

where $\sum_{q}$ runs over all quark and antiquark flavors, and $x$ and $x^{\prime}$ are given in Eq. (止).

To be consistent with our leading order calculation in $\alpha_{s}$, we use leading order parton distributions CTEQ5L for the numerical evaluation of the ratio $R_{A B}(Q)$ [13. Although the value of the $\lambda^{2}$ is not well fixed, we will use $\lambda^{2}=0.01 \mathrm{GeV}^{2}$ to calculate the $R_{A B}(Q)$, because it was extracted from a similar process - Drell-Yan transverse momentum broadening. The uncertainty in the value of $\lambda^{2}$ corresponds to a shift in overall normalization of the power corrections. we present the ratio $R_{A B}(Q)$ as a function of dilepton's invariant mass $Q$ in Fig. 3. The solid line is for $\mathrm{Pb}-\mathrm{Pb}$ collision at $\sqrt{S}=17.8 \mathrm{GeV} /$ nucleon with c.m. rapidity coverage $0<y_{c m}<1$, while the dashed line is for $\mathrm{p}-\mathrm{W}$ collision at $\sqrt{S}=30 \mathrm{GeV} /$ nucleon with c.m. rapidity coverage $-0.52<y_{c m}<0.48$.

From the dashed line in Fig. 3, the nuclear enhancement to the Drell-Yan continuum in the $p-W$ collision can be as large as $20 \%$ at $Q \sim 2 \mathrm{GeV}$. This is not in any contradiction 
with the data on the opposite-sign dilepton production from NA38 and NA50 collaborations, which show a good agreement with the expectation from all sources [四]. As shown in Ref. [4], a majority of lepton pairs in this IMR in hadron-nucleus collisions at $\sqrt{S}=30 \mathrm{GeV}$ come from open charm $D \bar{D}$ decay. Actually, a simultaneous fit of the 4 set $p-A$ data shows that the ratio of opposite-sign dileptons from the open charm decay and those directly from the Drell-Yan process is as large as $4.2 \pm 0.9$ at $\sqrt{S}=30 \mathrm{GeV}$ [4]. Therefore, a $20 \%$ increase of lepton pairs from the nuclear enhancement of the Drell-Yan continuum corresponds to less than $5 \%$ uncertainty in lepton pairs from $D \bar{D}$ decay, which is within the error bar of the fitting.

On the other hand, as shown in Fig. 9 of Ref. [4], the expected opposite-sign lepton pairs from the $D \bar{D}$ decay are less than the pairs from the Drell-Yan continuum in the $\mathrm{Pb}-\mathrm{Pb}$ collision. This is because total production rate of open charm $D \bar{D}$ pairs are much more suppressed than that of the Drell-Yan continuum when the collision energy decreases from $\sqrt{S}=30 \mathrm{GeV}$ for $p-A$ to $\sqrt{S}=17.8 \mathrm{GeV}$ for the $\mathrm{Pb}-\mathrm{Pb}$ collision. With the relative dominance of the Drell-Yan continuum in this IMR, a $50 \%$ enhancement of the Drell-Yan continuum at $Q \sim 2 \mathrm{GeV}$ from the solid line in Fig. 3 is a very significant change for the expected dilepton sources. Because of the mass threshold for producing open charm $D \bar{D}$ pairs, we expect the power corrections to the dilepton source from open charm decay to be much smaller.

We also emphasize that when the dilepton's invariant mass $Q$ is much less than $2 \mathrm{GeV}$, the leading order power corrections, as shown in Fig. 3, become so large that higher order power corrections have to be considered for a reliable QCD prediction. In addition, there should be high order corrections in $\alpha_{s}$ to the leading power contributions as well as the power corrections. Since we are interested in the relative size of these two contributions, we expect the ratio $R_{A B}(Q)$ to be relatively stable when we add high order corrections in $\alpha_{s}$. Finally, we point out that sign of the power corrections is not necessary to be positive as what we find here. Actually, as shown in Ref. [23], the power corrections to the longitudinal and transverse structure functions in deeply inelastic scattering carry an opposite sign.

In conclusion, we calculated the nuclear size enhanced power corrections to the inclusive Drell-Yan cross section in the IMR in hadron-nucleus and nucleus-nucleus collisions. We find that the medium size enhanced power corrections significantly enhance the dilepton production in the IMR, in particular, in the $\mathrm{Pb}-\mathrm{Pb}$ collision. Therefore, in order to understand the measured nuclear enhancement of lepton pairs in the IMR at CERN SPS, a new fit for the expected sources including the power corrections to the Drell-Yan continuum will be very important.

This work was supported in part by the U.S. Department of Energy under Grant Nos. DE-FG02-86ER40251 and DE-FG02-87ER40731. 


\section{REFERENCES}

[1] D. M. Alde et al., E772 Collaboration, Phys. Rev. Lett. 64, 2479 (1990).

[2] M.C. Abreu et al., NA50 Collaboration, Phys. Lett. B410, 327 (1997).

[3] M.C. Abreu et al., NA50 Collaboration, Phys. Lett. B410, 337 (1997).

[4] M.C. Abreu et al., NA38 and NA50 Collaborations, Eur. Phys. J. C14, 443 (2000).

[5] C. Y. Wong and Z. Q. Wang, Phys. Lett. B 367, 50 (1996).

[6] R. Rapp and E. Shuryak, Phys. Lett. B 473, 13 (2000).

[7] Z. Lin and X. N. Wang, Phys. Lett. B 444, 245 (1998).

[8] C.S. Lam and W.-K. Tung, Phys. Rev. D18, 2447 (1977).

[9] J.-W. Qiu and X.-F. Zhang, Phys. Rev. D64, 074007 (2001).

[10] J.C. Collins, D.E. Soper, and G. Sterman, in Perturbative Quantum Chromodynamics, edited by A. H. Mueller (World Scientific, Singapore, 1989), and references therein.

[11] S.D. Drell and T.M. Yan, Phys. Rev. Lett. 25, 316 (1970).

[12] G. Sterman et al., CTEQ Collaboration, Rev. Mod. Phys. 67, 157 (1995).

[13] H.L. Lai et al., Eur. Phys. J. C12, 375, (2000).

[14] J.-W. Qiu and G. Sterman, Nucl. Phys. B353, 137 (1991).

[15] J.-W. Qiu and G. Sterman, Nucl. Phys. B353, 105 (1991).

[16] J.-W. Qiu and G. Sterman, in preparation, and references therein.

[17] J. Qiu, Phys. Rev. D42, 30 (1990).

[18] D. Boer and J.-W. Qiu, hep-ph/0108179.

[19] A.H. Mueller and J.-W. Qiu, Nucl. Phys. B268, 427 (1986).

[20] M. Luo, J.-W. Qiu, and G. Sterman, Phys. Lett. B279, 377 (1992); Phys. Rev. D49, 4493 (1994).

[21] X.-F. Guo, Phys. Rev. D58, 114033 (1998).

[22] D. Naples et al., E683 Collaboration, Phys. Rev. Lett. 72, 2341 (1994).

[23] X.-F. Guo, J.-W. Qiu, and W. Zhu, in preparation. 


\section{FIGURES}
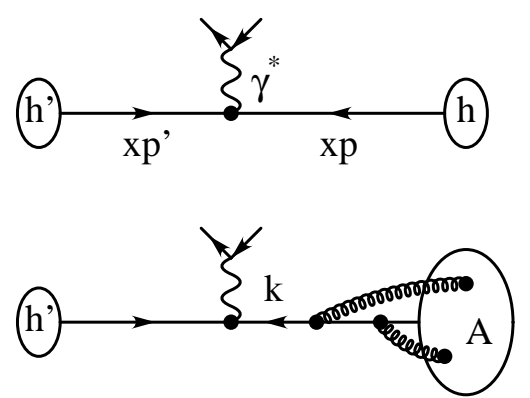

(b)

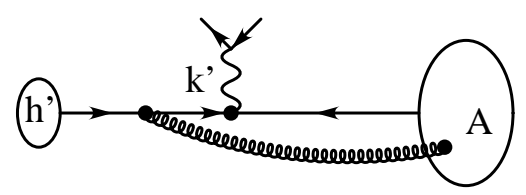

(c)

FIG. 1. Sketch for Drell-Yan process in hadron-hadron and hadron-nucleus collisions: (a) single hard scattering, (b) multiple interaction internal to the nucleus, (c) multiple interaction affects the hard scattering.

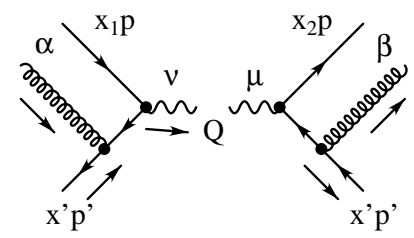

(a)
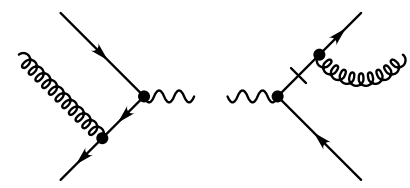

(c)
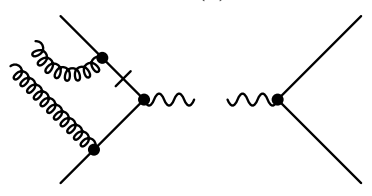

(g)
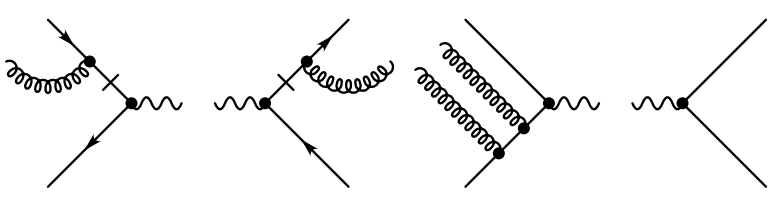

(b)
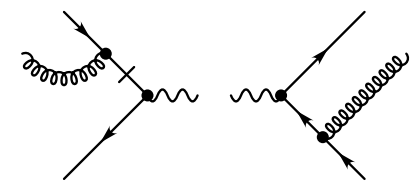

(d)
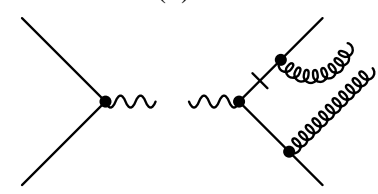

(h)

FIG. 2. Lowest order Feynman diagrams contribute to the leading power corrections to Drell-Yan cross section. 


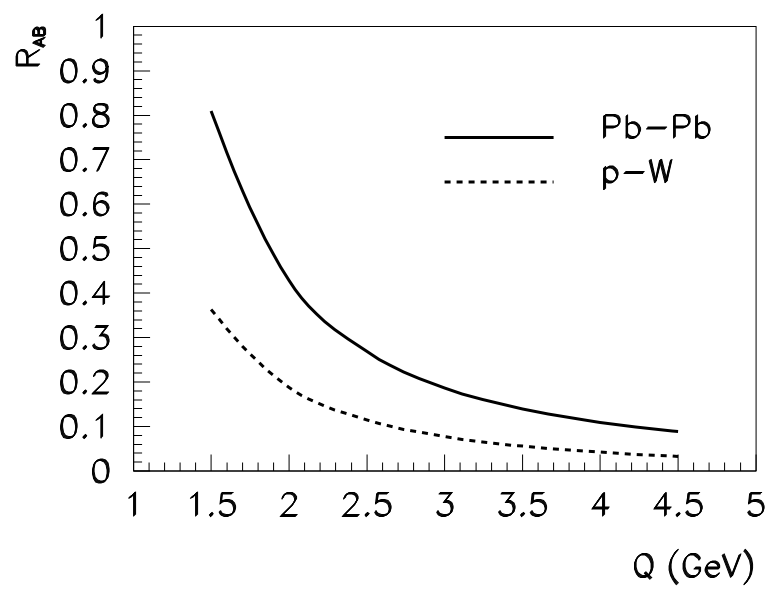

FIG. 3. The ratio $R_{A B}(Q)$ in Eq. (6) as a function of dilepton mass $Q$ in the $\mathrm{Pb}-\mathrm{Pb}$ (solid) and $p-W$ (dashed) collisions. 